\title{
TOWARDS IMPROVED ORGANISATIONAL SUPPORT FOR NURSES WORKING \\ IN RESEARCH ROLES IN THE CLINICAL SETTING. A MIXED METHOD \\ INVESTIGATION.
}

\begin{abstract}
Background: The clinical research workforce within nursing is growing including those employed to lead studies, coordinate research and many hybrid roles. Several studies have reported high job satisfaction among research nurses. However, there have also been reports of limited options for career development and professional integration, likely reflecting typical informal, departmentally-based management models. Institution-wide studies of issues related to research nurses are lacking, thus hampering the design and implementation of effective organisational frameworks to support and develop these positions. Aims: To explore experiences of nurses employed in research positions regarding organisational structures and support for research career pathways, and determine what reforms would strengthen an effective research specialisation pathway. Methods: A mixed-methods, cross-sectional approach, using a 104-item survey and semi-structured interviews of 11 staff in research roles at an acute care hospital in Queensland, Australia. Results: Research nurses lack organisational support in many job aspects that they deem important. A management model for the coordination of research nurses within a health district could maximise development of this field. Academic liaison and mentoring for nurses in research, and recognition for effort, are key areas for a management model to target. Conclusion: Nurses in research roles need individual mentorship, collective support, and the professional recognition and status that researchers in other settings are afforded. A comprehensive research management model would provide structured organisational support for nurses in research, improve professional development opportunities, ensure efficient use of human resources, synergistic working partnerships, and further contribute to a culture of evidence-based healthcare.
\end{abstract}


Keywords: Nursing Administration; Organisational research; Research Nurses; Clinical Nursing Research; Clinical Trial Coordinators 


\section{Introduction}

The demand for evidence-based practice in the health sector has resulted in a dramatic increase in the number of nurses involved in research employment, both in Australia (Australian Institute of Health and Welfare, 2008) and internationally (U.K. Clinical Research Collaboration, 2006). The Australian Nursing and Midwifery Labour Force Survey reports that one per cent $(>3,000)$ of registered nurses are employed as researchers in their primary job (this is in addition to those employed as academics), with others having a second job in research, and this has increased by $21 \%$ in the last five years (Australian Institute of Health and Welfare, 2008). A diverse array of opportunities exists for nurses interested in pursuing a research career, including pharmaceutical trials, managing audits and data registries, nursing research, multidisciplinary research, and academia (Raja-Jones, 2002; Rickard, Roberts, Foote \& McGrail, 2007). Modern healthcare facilities feature nurses working across all of these areas. However, little is known about how these positions are best integrated and managed from an organisational level, and how best to support and develop the skills of these nurses. This has major implications for the future planning and management of this valuable and untapped segment of the workforce.

Defining the careers of nurses in research is complicated by the abundance of position titles: Research Assistant, Research Nurse, Research Fellow, Research Coordinator, Trial Coordinator, Nurse Researcher and so on. We use the terms 'nurses in research', 'research nurses' and 'nurse researchers' interchangeably in this paper. There is great ambiguity regarding the role of nurses employed in research and significant problems in the way these positions are integrated into the health sector. Nursing employment arrangements are governed by a range of industrial agreements which vary in their inclusion of research designations. For example, the current Queensland Health award specifies a "Nurse Researcher” classification at Grade 7 without other junior or senior research classifications 
that would explicitly guide career progression (Queensland Health, 2009). In reality, many nurses are employed in research at (lower) grades 5 and 6, with others employed under professional officer, or higher education sector awards.

Clinical governance of research is increasingly regulated via the international 'Good Clinical Practice' guidelines, the National Statement on Ethical Conduct in Human Research, privacy legislation and local policies (Therapeutic Good Administration, 2000; National Health and Medical Research Council, 2007). Nurses in research require significant knowledge and expertise in these areas, for example, in adverse event monitoring and ensuring compliance with privacy legislation. Traditionally there has been a move to divide the roles of nurses in research into those who 'assist' rather than 'lead' research (e.g., coordinate trials designed by a pharmaceutical company or a medical/nursing researcher, rather than lead their own research as the chief investigator) (Rickard \& Roberts, 2008). In truth, the majority of these positions are now multi-faceted, with roles increasingly blurred and with similar/overlapping skill sets. For example in previous studies, one-third of research nurses were leading their own studies, in addition to managing those of others (Roberts, Eastwood, Raunow, Howe, \& Rickard 2011a).

Similar to clinical nursing work, most research nurse roles feature both collaborative activities in a multidisciplinary team, as well as autonomous responsibilities, with daily tasks including simple and complex activities (Raja-Jones, 2002). However, the transition from clinician to clinical research nurse is frequently made without clear position descriptions and to 'unofficial' research appointments (U.K. Clinical Research Collaboration, 2006). Wide variability has been reported in remuneration packages, organisational structure, and job security (Roberts \& Rickard, 2005). There appear to be common skills and responsibilities within these roles around the protection of subjects in research, the assurance of accurate data, excellent communication and organisational skills with a patient advocate and teaching 
function (Spilsbury et al. 2008; Roberts et al. 2011b). As research nurses experience increases their roles may expand unofficially, with one study finding that research nurses of more than 10 years' experience tended to additionally undertake activities such as statistical analysis, writing for publication, and presenting at conferences, yet without associated advancement in employment conditions or recognition (Roberts et al. 2011a). In 2006, a report on clinical trials in Australia identified a shortage of research nurses as a key issue constraining trial activity and noted the lack of a defined career path and pay scales that recognise their unique expertise (Pharmaceuticals and Biotechnology Branch, 2006).

The benefits and drawbacks of the nurse researcher role have been well documented. Studies have consistently observed high job satisfaction in terms of research nurses' interest in research itself, the ability to work autonomously, and to use their clinical nursing knowledge and skills (Mueller \& Mamo, 2002; Roberts, Rickard, Foote \& McGrail, 2006). Drawbacks and barriers to research work have been noted as isolation, lack of respect and recognition from nursing and medical colleagues, lack of career advancement opportunities, excessive workloads, insufficient funding for resources and poor remuneration (Castledine, 2001; Rickard et al., 2007; Spilsbury et al. 2008).

Although it has been acknowledged that supporting nursing research students and early career nurse researchers is vital to develop a program of nursing research (Emden \& Borbasi, 2000), the educational pathway for nurses to become involved in clinical research is not yet well structured in Australia. Undergraduate exposure to research traditionally involved theoretical methods-based instruction (Ecklund, 1999). This approach did not adequately prepare nurses to undertake randomised clinical trials and other clinical research studies. However, with nurse education now in the university sector, ward nurses are generally more amenable to the idea of a research career (Gill, 2004). Many research nurses pursue postgraduate research qualifications with 5-12\% studying at doctoral level in some studies 
(Rickard, Roberts, Foote and McGrail, 2006; Roberts et al. 2011a). The U.K. Clinical

Research Collaboration report (2006) recommended that strategy and infrastructure be made accessible to assist their research nurse career development from the outset, and encourage greater opportunity for academic training to doctoral level.

Research leadership roles and support structures have been found to vary considerably. Fitzsimons, McCance, and Armstrong (2006) discovered in Northern Ireland that few institutions had in place a strategy for the development of nursing and midwifery research. Conversely, those institutions with effective research leadership had correspondingly positive results, such as nurses and midwives having membership of advisory panels, senior boards and councils, and also input into the identification of strategic research priorities (Fitzsimons et al., 2006). The need for guidelines and policies that support nurses’ research has long been appreciated by overseas health departments (U.K. Department of Health \& Higher Education Funding Council, 2001). Formal support structures for nurse researchers are lacking in many Australian hospitals, and a research career structure is not explicit within nursing industrial awards, in contrast to other specialties such as nursing education or management. Nurses in research are frequently employed outside of the nursing support framework enjoyed by others such as clinical nurses or nurse educators, and often experience isolation and frustration in their roles (Mueller \& Mamo, 2002). Nurses in research require mentorship and individual support, and it is important to ensure that their research is applicable to clinical nursing (Schluter, Seaton \& Chaboyer, 2008). Given the recent move to national registration in Australia (Australian Nursing \& Midwifery Council, 2009), which sees formalised standards for registration and continuing practice, the need for such mentoring and supervision of nurses in all research roles is especially pertinent.

This current study targeted a large health service district in South-East Queensland, Australia and accessed personnel occupying research roles (however titled) within facilities 
there. This health district was chosen because, although there had been an increase in the number of nursing research roles there in the past decade, management structures and employment arrangements had not been altered. The study sought to understand the current experience of nurses in research roles in light of the current disjointed and disparate management models typical in Australia. In addition, we aimed to glean important information about how the situation might be better coordinated for the benefit of research nurses, researchers, patients, and the organisation as a whole. The satisfaction and importance that nurse researchers ascribe to their role is essential to identify strategies for their recruitment and retention (Price, 2002).

The aims of this study were to: 1 . explore the current experience of nurses employed in research positions in terms of the organisational structures and support for research nurse career pathways; and 2. to determine what reforms would be required to develop a clear and effective research specialisation pathway for nurses.

\section{Methods}

This study employed a mixed-method, cross-sectional approach involving a survey followed by a semi-structured, one-to-one interview that was conducted at the participating hospital at a time most convenient to the interviewee. Prior to the study there was no database of nurse researchers within the study setting; a regional general hospital of approximately 500 beds. A small group of nurses in research positions was identified and, using a snowball sampling technique, they were asked to forward the study details to others in similar, research-related roles. This approach was chosen, as opposed to the enforcement of a stringent inclusion and exclusion criteria, to reliably reflect the population of interest in a field with small numbers of representatives (Polit \& Beck, 2007). Participants were given the address for a secure internet website containing a questionnaire. Participation in completing the questionnaire was 
voluntary and anonymous, and computer IP addresses were not collected. Participants who completed the survey were also asked to contact the researchers if they agreed to be interviewed.

\section{The questionnaire}

The questionnaire contained three instruments: the Rickard-Roberts Research Coordinator Survey (RRRCS), the McCloskey-Mueller Satisfaction Scale (MMSS), and the McCloskeyMueller Importance Scale (MMIS). The RRRCS focuses on the role and responsibilities of research coordinators (Roberts et al. 2006). The MMSS (Mueller \& McCloskey, 1990), whilst originally developed for assessing job satisfaction of nurses working in the clinical setting, has been successfully used and validated with research nurses (Rickard et al., 2007; Roberts et al., 2006). The MMIS is an adaptation of the MMSS, seeing items rated for importance rather than satisfaction, and has also been used and validated with research nurses (Rickard et al., 2007; Roberts et al., 2006). All three questionnaires were modified slightly for an Australian research nurse cohort as described in Rickard et al. (2007). This included changes in wording from, for example, ‘faculty’ to ‘academics’ and ‘vacation' to ‘annual leave entitlements'.

In total there were 104 items, which took approximately 20-minutes to complete. Questions included: demographics; work history; aspects of the research role; current organisational structures; and the relative satisfaction and importance of employment conditions. The majority of questions required selection from predetermined options (drop down menu). Questions on satisfaction and importance of working conditions were answered using a 5-point Likert scale ranging from 'very dissatisfied/unimportant' to 'very satisfied/important'. Three free text sections (each a maximum of 150 characters) enabled participants to comment on the best and worst aspects of the role and comment further. 


\section{The semi-structured interview}

The audio-recorded interviews were semi-structured and undertaken by a universityemployed researcher who had no affiliation or involvement with the clinical services. Participants were asked about: career paths and aspirations; career structures; the profile of nursing research in the organisation; positive and negative aspects of the role; reporting and mentoring relationships; and the supports available. Interviewees were also asked their thoughts about a proposed organisational structure to coordinate and support nurses in research positions. The interviews took approximately one hour each.

\section{Ethical considerations}

The Gold Coast Health Service District and Griffith University Human Research Ethics Committees’ provided ethical approval for the study. The participant information and consent form clearly stated that participation was voluntary and that anonymity would be ensured in all reporting outputs.

\section{Data analysis}

The questionnaire data were tabulated with frequencies and percentages. Free text responses were clustered into themes and the incidence of each theme counted. The interviews were transcribed verbatim and were analysed thematically using a modified Colaizzi seven-step method (Colaizzi, 1978); a procedure for phenomenological data analysis that assumes a descriptive approach. This method of analysis was applied in this study by similar statements being grouped together into categories that were given a label. Categories were then examined for similarities and differences. Significant statements were extracted and meanings were formulated into themes. Two investigators analysed the data independently, then jointly reviewed the data to clarify categories and themes. 
The quantitative and qualitative data were considered in parallel when interpreting overall study findings and in developing recommendations.

\section{Results}

\section{Sample}

The lack of organisational recording of research nurse positions made it impossible to determine a response rate. Many participants were located by word of mouth. Eleven respondents completed the online survey: ten nurses and one physiotherapist (Table 1). This is consistent with previous studies, which show that while nurses fill the majority of clinical research positions (usually > 95\%) other health graduates, such as allied health or overseas trained physicians, also undertake these roles (Rickard et al., 2006). The ten nurses agreed to be interviewed.

\section{[Insert Table 1]}

The typical respondent was a female parent, in her 40s, with a decade or more of clinical experience in addition to research work history. Half had been in the current research position for one to three years, but many had also been employed in prior research roles. Eight nurses held postgraduate qualifications; two held a $\mathrm{PhD}$ and one was currently enrolled in a PhD program. Six nurses worked full-time in the research role, while others combined clinical or non-clinical nursing with research work.

\section{Moving into, and staying in, a research role}

Research employment opportunities in research were generally found to have occurred through word-of-mouth. Interviewees frequently reflected their feelings of good fortune or chance that they had become aware of the opportunity to specialise in research. Although most had not planned a career in research, others were inspired to apply for research positions 
after university studies had piqued their interest:

I did a small (names clinical specialty) course in Brisbane and my boss actually asked me if I would be interested in doing a ... study and that was 10-11 years ago. I have never had any problems getting a position in research.... It is word of mouth really... (NR10)

Most respondents indicated that they wished to remain in the research field. Consistent with the aforementioned increase in research positions, just over half were the first researchers to occupy their current position. Becoming a researcher was difficult for several respondents due to the lack of clear career pathways:

[Going into research] is not really a natural step because [there] has to be an opening there for nurses to step into. If it wasn’t there I would still be on the ward and you would see things that need changing and you wouldn't know the process about how to go about it. (NR2)

\section{Current role and responsibilities}

Survey respondents were asked about their current role, tasks and responsibilities (Table 2). One-third reported involvement in each of the following: departmentally initiated nursing research; pharmaceutical trials; and audits/data registries. Half reported involvement in departmentally initiated medical research or leading their own research. One-fifth were involved in 'other' research activities. These categories were not mutually exclusive, and respondents typically worked concurrently in several research areas. Most performed clinical research activities such as ethics submissions, data collection, and liaising with the health care team about research protocols. Half to two-thirds of the nurses engaged in more advanced research duties such as presenting at conferences and writing journal articles. Only one or two 
respondents were involved in activities such as chairing research committees or as a member of the ethics committee. Research was almost totally clinical in focus, with only two respondents reporting laboratory work as part of their role.

[Insert Table 2]

\section{Satisfaction with, and importance of, aspects of the position}

The 31 items ranked for both satisfaction and importance were compared for their relative scores (Table 3). Factors deemed most important included opportunity to interact with universities and academics and level of control over work. Despite this, 50\% reported that they did not have a relationship with an academic department; of those that did, 75\% were connected to a nursing academic department. The least satisfactory areas were: childcare facilities at work; opportunities to be included as a departmental staff member; and recognition by medical staff. Only $11 \%$ of the sample officially reported to an academic supervisor, $22 \%$ to a nursing research department, 33\% to a combined medical and nursing clinical manager, and 22\% to a clinical nursing manager. Generally, the researchers did not have subordinates who reported to them. The relative satisfaction: importance scores can be used to identify priority areas for action. Of the 31 items, 21 ranked higher for importance than satisfaction, indicating areas of desired improvement. In 12 cases, this difference was marked, measuring at least one full point on the response scale.

\section{[Insert Table 3]}

Recurring themes in both the survey and interview responses to the 'best' aspects of working as a nurse researcher included:

- autonomy;

- diversity of the role with interesting work that was challenging and multifaceted; 
- family-friendly work hours;

- satisfaction derived from contributing to health knowledge, practice and the dissemination of research findings;

- ability to apply existing sound clinical knowledge in the area of research, and;

- satisfaction of working as part of the multidisciplinary team towards a common goal.

Several reported the importance of having 'an opportunity to contribute', 'being there for patients', and 'going the extra mile for them':

[As a nurse] you are always on the lookout for something better, a better process of doing something or better at making your client's ride through life better and making their health better or whatever it is ... research is the big step further. NR2

Recurring themes in both the survey and interview to the 'worst' aspects of working as a nurse researcher included:

- professional isolation;

- inadequate time, funding, and resources;

- lack of job security;

- lack of departmental and organisational support;

- lack of understanding and respect from colleagues, and;

- inadequate recognition of the researcher role and corresponding lack of professional standing

The survey item 'opportunity to be included as a departmental member' scored high for importance but low for satisfaction. Interview data confirmed that many felt isolated and 
undervalued and that research nursing careers are not sufficiently supported, neither financially nor through organisational structures:

Basically we are all on contract, so when that contract finishes if the money is there we continue on... If the money runs out our position runs out. (NR1)

One thing ... massively lacking is actual space, equipment and resources to go along with the position...I have tried to get funding to get (statistical software) that I actually need to do my job ... and I can’t get it. (NR7)

One rate-limiting factor was that supervisors and employing organisations reportedly did not see it as necessary to back-fill positions while incumbents were on leave. This was a stressor to the nurses who felt they were expected to maintain research standards and workloads even when they were on leave. This may be because most supervisors were clinical managers who did not have full insight into the research role. However, even those nurses with university-based managers reported that these academics did not always appreciate the complexity or workload of their clinical research positions.

\section{Educational preparation and ongoing education for the position}

Educational preparation for research varied considerably. While five nurses had postgraduate qualifications that had entailed research methods exposure, others had little or no formal training in research. 'Learning on the job’ was common, as also identified by Mueller and Mamo (2002). Having pre-existing, in-depth knowledge in the clinical specialty associated with the research was reported to be extremely important and helpful. Of the nurses who did not have any formal education in research, all agreed it would be useful to undertake a structured research educational course. Programs covering topics such as clinical trial 
management, data management, and compliance with regulatory and legislative requirements for research (e.g. ethics submissions, TGA requirements, privacy laws) would be seen as useful. However, it does not necessarily follow that all nurse researchers wish to pursue formal postgraduate studies to the point of designing their own studies.

Most were doubtful that undergraduate nursing education provided any beneficial research grounding. Becoming a nurse researcher entailed a 'steep learning curve' for many, and most sought a supportive mentor to facilitate the transition. Those who had completed postgraduate studies reported having a university mentor. Other mentors were usually other nurse researchers in the clinical area, nurse manager, nursing director, medical staff, and a pharmacist (for pharmaceutical trials). Several reported acting as mentors to junior nurse researchers or other nurses interested in pursuing a career in research.

The educational preparation for working as a nurse researcher varied for study participants. All nurses agreed that research education was valuable and important. For some, the area of research had sparked interest in obtaining postgraduate qualifications. Several had attended research courses or workshops, but reported that the quality of these varied considerably.

Only half of the sample had any relationship with an academic university department, and this was seen as a very valuable support. Most often this was with a nursing academic department:

Doing the PhD has helped, mainly because it has given me those underlying skills that I think I need in this particular role...[My supervisor] has been great in providing access for me to extra support... I was able to have the opportunity to work with [a statistician]... I have an adjunct position with the University research centre so that helps me because I am able to go to extra courses or lectures or anything like that. (NR7) 


\section{Organisational structures and networks}

The respondents identified existing supports, areas lacking support, and suggestions for improving research structure within the organisation. These are presented in Table 4.

\section{[Insert Table 4]}

On-the-job learning played a fundamental role in teaching nurse researchers about their position. All mentioned the importance of a supportive role model. Reported mentors included academic supervisors, nurse managers, nurse educators, medical directors, and pharmacists. Other important sources of knowledge and support included multidisciplinary team members, the local research committee, an existing nursing research group, organisational website, on-line organisations, and networking with other nurse researchers. Several mentioned the importance of having an organisational culture that supports research: Having a nursing director [who] is supportive of research is very important... If the organisation has a culture of research then you have a few people doing it and they show other people how to do it. It is sort of like learning on the job in a practical sense. (NR9)

\section{Ideas for improving organisational structures and support for clinical research positions}

Many nurse researchers cited isolation and lamented the lack of a formal research support structure. As a consequence, several had actively sought research support within their clinical division; however, some divisions were better set up than others to provide this support. Respondents reported several areas lacking in support for research. The lack of a clear research structure frustrated many. Access to information on research scholarships, courses or workshops was usually by word-of-mouth. Several researchers reported that they did not know other researchers in the district and that this would be helpful for networking purposes. 
Relying on research colleagues for support could also be problematic when they were too busy with their own research to provide assistance. Two researchers reported receiving limited or no research support. Despite this lack of support, many remained positive about the role and suggested ideas for improving the research structure:

I think it could be better; we need a structure... a person that is in charge of all the research nursing staff to actually report to the [director of nursing]...They need to have that structure there so rosters can be worked around if someone is sick, the admin side can deal with it and the clinician side of it can continue on and follow up. It is not unified... There is no good structure there to be able to guide [contract staff] and make sure they have the accredited (research) courses to continue on. (NR1)

Despite the reported difficulties of isolation and lack of support structure, most of the nurses interviewed said that, if given the opportunity again, they would choose research as a career: 'Definitely', 'I would have done it sooner'. Most reported that they planned to continue working in research for as long as possible. The majority also reported encouraging other nurses to consider a career in research. Educating clinical nurses about research trials and study findings provided opportunities for these nurse researchers to share their enthusiasm for the role.

\section{Discussion}

A recurrent theme that emerged from both survey and interviews was the need for improved recognition of, and consultation with, nurses employed in research. Professional isolation was cited repeatedly, as was a perceived lack of understanding and respect from colleagues. Previous studies have also highlighted these issues (Castledine, 2001; Rickard et al., 2007; Roberts et al., 2006), thus suggesting that there is a strong need for nurses working in clinical research to feel valued and to be consulted about their expertise. The findings also 
highlighted the need for clinical research roles to be promoted and acknowledged within the profession and also within pre-registration education. In line with previous studies, nurse researchers reported high levels of overall job satisfaction, despite it being a career choice that was primarily driven by chance and opportunity (Mueller \& Mamo, 2002; Roberts et al., 2006). Promotion of a clearer research career pathway within nursing is evidently necessary.

As in previous research, the employment and management of research nurses was highly variable between clinical departments and there was great disparity in contract conditions and reporting requirements (Roberts \& Rickard, 2005). Any supportive or professional networking between the nurses had generally happened at their own initiative, and performance management or professional development was usually ad hoc and 'on-the job’, as reported by previous authors (Mueller \& Mamo, 2002). A lack of organisational support was highlighted as a barrier in role development and function, thus indicating that organisational reform, and a superior management model, is required to better support structures for new and existing nurse researchers. Findings suggest that the management model should target:

- inclusiveness within the department and organisation. The fostering of better interdisciplinary interaction, to facilitate role recognition from management and clinical nursing and medical staff. Promotion of social contact with colleagues within the workplace;

- academic interaction and affiliation with universities and university-based researchers;

- improved researcher control over working conditions including the implementation of feedback facilities for the researchers. Improved researcher involvement with overall research processes in the department/institution rather than only at project level.

- human resource management and industrial award particulars, including role category, description, and remuneration. Consideration regarding the employment of research 
nurses on longer-term contracts and at appropriate levels and designations;

- implementing structure and policy for research career advancement, and access of relevant educational programs

As in previous research, nurses highlighted the importance of a supportive role model for mentorship and individual support (Schluter et al., 2008). For the majority of clinical nurses there is a single point operational and professional manager (i.e. clinical line manager). This study found that most nurses in research positions typically were also officially managed at the clinical departmental level (by clinical medical or nursing managers), rather than by a senior researcher or research manager. In light of this, it may be advantageous for organisations to have a designated senior leader for all nurses employed in research. An institutional manager with responsibility to support and develop nurses in research (N.B. not nursing research per se) would be a clear benefit. The position would provide a championing role at senior level, and a channel for new and experienced research nurses to access information and guidance in their roles and careers, with the manager being aware of all nurses working in research roles throughout the institution. Research coordinator networks and regular seminars have been found to provide team cohesion and support (Ecklund, 1999; Richardson, Turnock, \& Gibson, et al., 2007; Spilsbury et al., 2008), and this may be particularly important to assist with building networks that have practical and professional benefits (e.g. leave arrangements, mentoring).

Previous research has identified the importance of orientation, mentoring and continuing education for nurse researchers (Schluter et al., 2008). However, in this study, few nurses reported any initial orientation or mentoring, and this was overwhelmingly suggested as an area for improvement. Respondents were enthusiastic about regular seminars for ongoing development and greater efforts should be undertaken to harness this enthusiasm for 
continuing education. Encouragement and support is also needed for those research nurses who wish to continue on to higher degrees or postgraduate qualifications in research, as reflected in the U.K. Clinical Research Collaboration's (2006) education and training recommendations (i.e., establishment of a range of coordinated research training opportunities and annual funding of clinical academic training positions). In addition, although the surveyed institution already had strong links to nursing and other academic departments, most respondents desired stronger links with universities. Half currently had an official adjunct title or collaborated with a university department, but most felt increased engagement with universities would lead to more efficient research, and more thorough dissemination of research findings. Previous research highlights research nurses’ interest in developing the academic aspects of research such as publishing and presenting at conferences outweighs their confidence in doing so (Roberts et al. 2011b) We suggest that one role of the mentoring and professional management provided a central research nurse manager, would be to work with the nurse to identify appropriate mentors and collaborators from the academic sector.

Finally, although the nurses in this study did not specifically mention lack of remuneration for on-call work as found to be a concern previously (Rickard et al. (2007); Roberts et al. (2011b) several mentioned that lack of funding made it difficult to obtain essential resources, such as computers and adequate workspaces. Access to computers, research-specific software, and private, dedicated workspaces is a basic requirement of a role that requires secure data entry for confidentiality. Other researchers expressed concern that when the funding for studies dried up, their own positions would be terminated. A clearer organisational structure and career pathway may go some way to alleviate concerns regarding job security and ensure nurse researchers have access to the essential resources they need to conduct research. An alternate model would be for Nursing Directors to establish a Department of Clinical Trials/Research through which to employ and support research nurses, 
and for the clinical investigators and departments to access the nurses' services on a fee-for service (cost-recovery) model. This would not only allow the research nurses to have greater job security and support, but for the clinical investigators to be relieved of some of the administrative burden as an employer/manager, and instead focus on the research and their clinical duties. We stress that this arrangement would require a strong 'customer service' focus on the clinical researchers and departments, or they would likely return to the current ad hoc arrangements.

\section{Practice implications and recommendations}

After analysis of both the quantitative and qualitative data and consideration of prior research in this area, a number of recommendations can be made to encourage greater nurse researcher support in clinical organisations:

1. Improved recognition of, and consultation with, nurses employed in research: further research should be undertaken to determine what organisational models best facilitate nurses to manage research (their own and others), and also how current organisational and professional structures do (and do not) facilitate the optimal development of such positions.

2. Promotion of the clinical research nurse role as a rewarding potential career choice: careers advisors should be made aware of the range of opportunities available to qualified nurses, and nursing students should have greater exposure to clinical research (both nursing led and interdisciplinary) through lectures, practicums and summer placements.

3. Organisational reform within clinical facilities: a superior management model should be developed, in consultation with research nurses and key stakeholders such as clinical researchers, and academic partners. 
4. Provision of a designated senior leader for nurses employed in research: the employment of an institutional manager with responsibility to support and develop research nurses (not nursing research per se) should be considered. Consultation with research nurses and employing departments would be needed to determine the line of reporting.

5. A focus on orientation, mentoring and continuing education: could include buying-in training programs offered by external companies, accessing affiliated academic or senior local researchers and operating a monthly seminar series or twice yearly professional development day for all nurses employed in research.

6. Increased engagement with the academic sector: the proposed central manager could facilitate the process of collaboration and mentorship with the academic sector. Consultation with nurse academics on how to strengthen collaborations between universities and hospitals would be advantageous.

7. Greater access to resources (e.g. computers, research software, private workspaces): funds should be allocated to ensure the research nurse has access to at least a computer, space for confidential telephone calls and meetings, Storage space for confidential documents, and research-specific computer software to allow them to do their job.

Successful implementation of these recommendations would most likely be achieved through the establishment of an implementation group. This group would ideally comprise key stakeholders whom have the direct authority to action change and implement the recommendations, such as relevant individuals from the public and private sector employers, industrial bodies, the higher education sector, the Department of Health and Ageing, the National Health and Medical Research Council, the Royal College of Nursing Australia and 
the Australian Nursing \& Midwifery Council. A review of the implementation would be necessary bi-annually to ensure progress and also flexibility in approach, given changes in the political and economic climate.

\section{Limitations}

The study had a small sample size $(n=11)$ and included nurse researchers from only one hospital in South-East Queensland, Australia. This limits the generalisability of the results and also permits any definitive recommendations regarding the skill set required for nurses in research nurse, or mapping of role and functions to competency standards (Australian Nursing and Midwifery Council, 2006). However, the 'whole of hospital' approaches provides a more comprehensive case study of this phenomena than other works, which focus on particular specialty areas, and many issues raised are consistent with prior studies in the area (Mueller and Mamo, 2002; Rickard et al., 2007).

\section{Conclusion}

Increasing numbers of nurses are attracted to working in research roles, despite a general lack of formal organisational structures to support research careers and networks for nurses employed in these roles. This is an untapped and undervalued sector. Whilst overall job satisfaction is high, restructuring and recognition could better support these positions and maximise their potential to contribute to the profession and to patient care. Nurse researchers need mentorship, individual support, and the professional validation and status that researchers in other settings are afforded. Providing better, more structured, organisational support for nurse researchers is likely to maximise their contribution to the overall research and evidence-based culture and productivity within health care institutions. 
Table 1 Demographic data (from online survey)

\begin{tabular}{|c|c|c|}
\hline Item/category & Descriptor & $n$ \\
\hline \multirow[t]{2}{*}{ Gender } & Female & 8 \\
\hline & Male & 2 \\
\hline \multirow[t]{3}{*}{ Age } & $31-40$ & 2 \\
\hline & $40-50$ & 8 \\
\hline & $51-60$ & 1 \\
\hline \multirow{5}{*}{ Highest current qualification } & Hospital certificate & 2 \\
\hline & Graduate diploma & 1 \\
\hline & Masters (coursework) & 3 \\
\hline & Masters (research) & 2 \\
\hline & $\mathrm{PhD}$ & 2 \\
\hline \multirow[t]{3}{*}{ Time in clinical role (years) } & $1-3$ & 1 \\
\hline & $7-9$ & 3 \\
\hline & $>9$ & 5 \\
\hline \multirow[t]{4}{*}{ Time in current research position (years) } & $<1$ & 2 \\
\hline & $1-3$ & 5 \\
\hline & $7-9$ & 1 \\
\hline & $>9$ & 1 \\
\hline \multirow[t]{2}{*}{ Employment status } & Full-time & 6 \\
\hline & Part-time & 4 \\
\hline \multicolumn{3}{|c|}{ Is respondent first person to occupy current } \\
\hline \multirow[t]{6}{*}{ Area of research ${ }^{\mathrm{a}}$} & Pharmaceutical research & 4 \\
\hline & Departmental medical research & 6 \\
\hline & Departmental nursing research & 3 \\
\hline & Own research & 5 \\
\hline & Audits/data registries & 3 \\
\hline & Other & 2 \\
\hline
\end{tabular}

Note. ${ }^{\text {a }}$ Some identified more than one area.

Missing data in some fields (some respondents did not answer every question). $n=11$ comprised of 10 nurses \& 1 physiotherapist. 
Table 2 Tasks conducted by clinical researchers (from online survey)

\begin{tabular}{lll}
\hline Item description & $n$ & $\%$ \\
\hline Liaising with the health care team & 9 & 81 \\
Data collection & 9 & 81 \\
Ethics submissions & 9 & 81 \\
Providing research advice and teaching & 8 & 72 \\
Obtaining consent & 8 & 72 \\
Data entry & 8 & 72 \\
Literature searches & 7 & 63 \\
Designing data collection tools & 7 & 63 \\
Member of nursing research committee & 7 & 63 \\
Presenting at conferences & 7 & 63 \\
Assessing patients & 6 & 54 \\
Grant submissions & 6 & 54 \\
Organising research meetings & 6 & 54 \\
Clinical research procedures & 5 & 45 \\
Reviewing protocols & 5 & 45 \\
Writing for publication & 5 & 45 \\
Protocol development & 5 & 45 \\
Providing patient/relative education and support & 4 & 36 \\
Statistical analysis & 4 & 36 \\
Database design & 4 & 36 \\
Laboratory research & 2 & 18 \\
Chair research meetings & 1 & 9 \\
Member of ethics committee & 1 & 9 \\
\hline Note. n-11 comprsed 10 nurses $\&$ physiotrats & & \\
\hline
\end{tabular}

Note. $n=11$ comprised of 10 nurses \& 1 physiotherapist. 
Table 3 Mean importance versus mean satisfaction scores (from online survey)

\begin{tabular}{|c|c|c|c|}
\hline Item description & $\begin{array}{l}\text { Mean } \\
\text { Satisfaction }^{1}\end{array}$ & $\begin{array}{l}\text { Mean } \\
\text { Importance }^{1}\end{array}$ & Difference \\
\hline \multicolumn{4}{|l|}{ More important: less satisfied } \\
\hline $\begin{array}{l}\text { Opportunity to be included as a departmental } \\
\text { member }\end{array}$ & 2.6 & 4.9 & -2.3 \\
\hline $\begin{array}{l}\text { Opportunity to interact with } \\
\text { universities/academics }\end{array}$ & 2.9 & 5.0 & -2.1 \\
\hline Child care facilities at work ${ }^{\mathrm{c}}$ & 1.0 & 3.0 & -2.0 \\
\hline Encouragement and feedback in this position & 3.0 & 4.6 & -1.6 \\
\hline Salary level & 3.0 & 4.4 & -1.4 \\
\hline Recognition from medical staff ${ }^{\mathrm{b}}$ & 2.6 & 4.0 & -1.4 \\
\hline Level of control over working conditions & 3.6 & 4.9 & -1.3 \\
\hline Social contact with colleagues at work & 3.6 & 4.9 & -1.3 \\
\hline Involvement with overall research processes & 3.3 & 4.4 & -1.1 \\
\hline Opportunity for career advancement ${ }^{\mathrm{a}}$ & 3.0 & 4.1 & -1.1 \\
\hline Level of control over work & 4.0 & 5.0 & -1.0 \\
\hline Level of input in decision making & 3.9 & 4.9 & -1.0 \\
\hline Recognition from nursing staff & 3.6 & 4.4 & -0.9 \\
\hline Non-salary aspects of remuneration package & 2.9 & 3.7 & -0.9 \\
\hline Level of responsibility in the position & 4.0 & 4.7 & -0.7 \\
\hline Compensation for weekends worked ${ }^{\mathrm{c}, \mathrm{d}}$ & 3.5 & 4.2 & -0.7 \\
\hline Annual leave entitlements & 4.1 & 4.7 & -0.6 \\
\hline Number of hours worked & 4.0 & 4.6 & -0.6 \\
\hline Opportunity to undertake own research ${ }^{\mathrm{a}}$ & 3.7 & 4.1 & -0.5 \\
\hline Satisfaction with immediate supervisor & 4.6 & 4.9 & -0.3 \\
\hline Opportunity to write/publish ${ }^{\mathrm{a}}$ & 3.8 & 4.0 & -0.2 \\
\hline \multicolumn{4}{|l|}{ Equal importance: satisfaction } \\
\hline Working with other researchers and research staff & 4.3 & 4.3 & 0.0 \\
\hline Working with medical staff & 3.6 & 3.6 & 0.0 \\
\hline Flexibility of your days of work & 4.6 & 4.6 & 0.0 \\
\hline Opportunity to interact with other disciplines & 4.7 & 4.7 & 0.0 \\
\hline \multicolumn{4}{|l|}{ More satisfied: less important } \\
\hline Opportunity to work normal business hours & 4.6 & 4.4 & 0.1 \\
\hline Number of weekends off per month & 4.9 & 4.7 & 0.1 \\
\hline Flexibility of weekends off, without on call ${ }^{\mathrm{d}}$ & 4.9 & 4.3 & 0.5 \\
\hline Opportunity to work part-time ${ }^{a}$ & 4.3 & 3.3 & 1.0 \\
\hline Opportunity for maternity/parental leave ${ }^{\mathrm{c}, \mathrm{d}}$ & 3.5 & 2.2 & 1.3 \\
\hline Social contact with colleagues outside of work & 4.6 & 1.9 & 2.7 \\
\hline
\end{tabular}

Note. $n=11$ comprised of 10 nurses \& 1 physiotherapist.

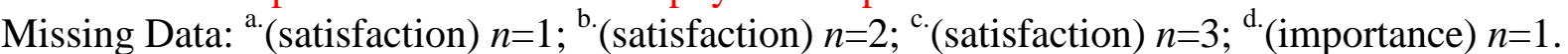

${ }^{1}$ Mean satisfaction and importance scores derived from a five-point Likert scale where $5=$ 'very satisfied/important' and 1 = 'very dissatisfied/unimportant'. 
Table 4 Existing supports, areas perceived to be lacking in support and ideas for improving the research structure (from interview data)

\begin{tabular}{|c|c|c|}
\hline Supports currently available and utilised & Perceived lack of support & Ideas for improving the research structure \\
\hline Supportive manager/supervisor (6 comments) & $\begin{array}{l}\text { Workload pressures, overwhelming/stressful at } \\
\text { times ( } 7 \text { comments) }\end{array}$ & Supportive role model/mentor (10 comments) \\
\hline Supportive organisation/employer (5 comments) & $\begin{array}{l}\text { Lack of funding for research nurses (5 } \\
\text { comments) }\end{array}$ & $\begin{array}{l}\text { Networking with other researchers (10 } \\
\text { comments) }\end{array}$ \\
\hline Supportive university mentor (4 comments) & $\begin{array}{l}\text { Lack of unity among researchers in other areas } \\
\text { (4 comments) }\end{array}$ & $\begin{array}{l}\text { Recognition of value of research as a career } \\
\text { choice ( } 8 \text { comments) }\end{array}$ \\
\hline Research committee (4 comments) & $\begin{array}{l}\text { Lack of unity between researchers \& clinicians } \\
\text { ( } 3 \text { comments) }\end{array}$ & Research education opportunities (7 comments) \\
\hline Supportive medical staff (4 comments) & Lack of local research support (3 comments) & Organisational culture of research (5 comments) \\
\hline Nursing research group (3 comments) & $\begin{array}{l}\text { Nursing research group poorly attended ( } 3 \\
\text { comments) }\end{array}$ & More funding (5 comments) \\
\hline Supportive nurse educator (3 comments) & Lack of job security (3 comments) & More resources (e.g. computers) (2 comments) \\
\hline Supportive multidisciplinary team (3 comments) & Chronic research staff shortage (3 comments) & $\begin{array}{l}\text { Paid time to attend conferences/workshops ( } 2 \\
\text { comments) }\end{array}$ \\
\hline Organisational website is valuable (2 comments) & No back up while sick/away (3 comments) & $\begin{array}{l}\text { Time for ward nurses to participate in research } \\
\text { projects ( } 2 \text { comments) }\end{array}$ \\
\hline Supportive pharmacist (1 comment) & $\begin{array}{l}\text { Clinical staff lack time to get involved in } \\
\text { research ( } 3 \text { comments) }\end{array}$ & $\begin{array}{l}\text { Time \& opportunity to maintain clinical nursing } \\
\text { skills ( } 2 \text { comments) }\end{array}$ \\
\hline $\begin{array}{l}\text { Support from pharmaceutical companies ( } 1 \\
\text { comment) }\end{array}$ & $\begin{array}{l}\text { Organisation/employer is unsupportive ( } 2 \\
\text { comments) }\end{array}$ & Time to reflect on practice (2 comments) \\
\hline $\begin{array}{l}\text { Online organisations have been helpful (1 } \\
\text { comment) }\end{array}$ & $\begin{array}{l}\text { Lack of respect from nursing staff ( } 2 \\
\text { comments) }\end{array}$ & Journal clubs (1 comment) \\
\hline & $\begin{array}{l}\text { Lack of respect from medical staff ( } 2 \\
\text { comments) } \\
\text { Lack of education and research resources ( } 2 \\
\text { comments) } \\
\text { Lack of computer and office resources ( } 2 \\
\text { comments) } \\
\text { Getting time off to attend conferences is }\end{array}$ & \\
\hline
\end{tabular}


difficult (2 comments)

Lack of paid time to do research (2 comments)

Research is not an organisational priority (1

comment)

Lack of opportunity for study leave (1

comment)

Lack of support from line manager (1

comment)

Need more administrative support (1 comment)

Difficult to involve staff in research when

manager is not supportive (1 comment)

Note. $n=10$ nurses were interviewed. 


\section{References}

Australian Institute of Health and Welfare. (2008). Nursing and midwifery labour force 2005.

Canberra: Australian Institute of Health and Welfare.

Australian Nursing \& Midwifery Council (2006). National competency standards for the registered nurse ( $4^{\text {th }}$ ed). Dickson, ACT: Australian Nursing \& Midwifery Council.

Castledine, G. (2001). Whatever has happened to nursing research? British Journal of Nursing, 10, 131.

Colaizzi, P.F. (1978). Psychological research as the phenomenologist views it. In R.S.Valle \& M. King (Eds.), Existential-Phenomenological Alternatives for Psychology (pp. 48-71). New York: Oxford University Press.

Ecklund, D.J. (1999). Establishing a research coordinator network: The birth of an idea. Journal of Nursing Administration, 29, 20-25.

Emden, C., \& Borbasi, S. (2000). Programmatic research. A desirable (or despotic?) nursing strategy for the future. Collegian, 7, 32-37.

Fitzsimons, D., McCance, T., \& Armstrong, N. (2006). Vision, leadership and partnership: How to enhance the nursing and midwifery contribution to research and development. Journal of Advanced Nursing, 55, 748-756. 
Gill, P. (2004). Difficulties in developing a nursing research culture in the UK. British Journal of Nursing, 13, 876-879.

Mueller, M.R., \& Mamo, L. (2002). The nurse clinical trial coordinator: Benefits and drawbacks of the role. Research Theory in Nursing Practice, 16, 33-42.

Mueller, C.W., \& McCloskey, J.C. (1990). Nurses’ job satisfaction: A proposed measure. Nursing Research, 39, 113-117.

National Health and Medical Research Council. (2007). National Statement on Ethical Conduct in Human Research. Australian Government, Canberra. p.1-107.

Pharmaceuticals and Biotechnology Branch, Department of Industry Tourism and Resources. (2006) Report on the Forum on a National Approach to Clinical Trials. Commonwealth of Australia. Canberra, ACT. p.1-32.

Polit, D.F., \& Beck, C.T. (2007). Nursing research: Generating and assessing evidence for nursing practice. Philadelphia: Lippincott Williams \& Wilkins.

Price, M. (2002). Job satisfaction of registered nurses working in an acute hospital. British Journal of Nursing, 11, 275-280.

Queensland Health. (2009). Nurses and Midwives (Queensland Health) Certified Agreement (EB7). 
Raja-Jones, H. (2002). Role boundaries - research nurse or clinical nurse specialist? A literature review. Journal of Clinical Nursing, 11, 415-420.

Richardson, A., Turnock, C., \& Gibson, V. (2007). Development of a critical care nursing research strategy: A tripartite approach. British Journal of Nursing, 16, 1201-1207.

Rickard, C.M. \& Roberts, B.L. (2008). Commentary on the role and potential contribution of clinical research nurses to clinical trials. Journal of Clinical Nursing, 17, 2664-2666.

Rickard, C.M., Roberts, B.L., Foote, J., \& McGrail, M.R. (2006). Intensive care research coordinators: Who are they and what do they do? Results of a binational survey. Dimensions in Critical Care Nursing, 25, 234-242.

Rickard, C.M., Roberts, B.L., Foote, J., \& McGrail, M.R. (2007). Job satisfaction and importance for intensive care unit research coordinators: Results from binational survey. Journal of Clinical Nursing, 16, 1640-1650.

Roberts, B.L., \& Rickard, C. (2005). The role of the ICU research coordinator in Australia: An invaluable resource for ICU research. Critical Care and Resuscitation, 7, 52-56.

Roberts, B., Eastwood, G.M., Raunow, H., Howe, B., \& Rickard C.M. (2011a). Intensive care research coordinators in Australia and New Zealand: A cross-sectional survey of demographics, responsibilities, job satisfaction and importance. Australian Critical Care. March 17. [epub ahead of print]. 
Roberts, B., Eastwood, G.M., Raunow, H., Howe, B., \& Rickard C.M. (2011b). The intensive care research coordinator position in Australia and New Zealand: Self-perception of professional development priorities and 'best and 'worst' aspects of the position. A crosssectional web-based study. Intensive and Critical Care Nursing.

Roberts, B.L., Rickard, C.M., Foote, J., \& McGrail, M.R. (2006). The best and worst aspects of the ICU research coordinator role. Nursing in Critical Care, 11, 128-135.

Schluter, J., Seaton, P., \& Chaboyer, W. (2008). Critical incident technique: A user's guide for nurse researchers. Journal of Advanced Nursing, 61,107-114.

Spilsbury, K., Petherick, E., Cullum, N., Nelson, A., Nixon, J., \& Mason, S. (2008). The role and potential contribution of clinical research nurses to clinical trials. Journal of Clinical Nursing, 17, 549-557.

Therapeutic Goods Administration. (2000). Note for Guidance on Good Clinical Practice (CPMP/ICH/135/95). Commonwealth Department of Health and Aged Care. Canberra.

U.K. Clinical Research Collaboration. (2006). Developing the best research professionals. Qualified graduate nurses: Recommendations for preparing and supporting clinical academic nurses of the future. London: U.K. Clinical Research Collaboration.

United Kingdom Department of Health and Higher Education Funding Council for England. (2001). Promoting research in nursing and the allied health professions: Report of Task 
Group 3 to HEFCE and the Department of Health (Research report 01/64). Bristol: United Kingdom Department of Health and Higher Education Funding Council for England. 